\title{
Labor profunda en siembra directa: Efecto sobre la condición biológica del suelo
}

\author{
Claudia Alzugaray, María Sofía Vilche y Cristina Petenello \\ Facultad de Ciencias Agrarias, Universidad Nacional de Rosario, Campo Experimental Villarino. C.C.14. \\ S2125ZAA. Argentina.
}

\begin{abstract}
C. Alzugaray, M.S. Vilche, and C. Petenello. 2008. Deep tillage in the no-till system: Effects on the biological condition of the soil. Cien. Inv. Agr. 35(3):265-276. The no-tillage system offers the advantages of greater stability and biological diversity, although it may generate soil compaction. This mechanical restriction can be solved with deep tillage, which is able to generate loosening that preserves the superficial covertures. The biological condition of soil is very sensitive to disturbances resulting from tillage. The objective of this study was therefore to evaluate changes in the biological condition of soil that are caused by the use of paratill under no-tillage lots. The experiments were conducted using corn crops in Zavalla, Argentina $\left(60^{\circ}\right.$ $53^{\circ} \mathrm{W}$ and $\left.33^{\circ} 01^{\prime} \mathrm{S}\right)$, in a vertic Argiudoll. The treatments included no-tillage and no-tillage with paratill. The following parameters were assessed: soil organic carbon, nitrates, sulfates and phosphorous; biomass and microbial respiration activity; meso- and macrofauna; root channels; sites with fungi and decomposed organic matter; krotovinas and root system depth at the fiveleaf stage of cultivation; and aerial and root dry matter. The data were processed by uni- and multivariate analysis. The decompaction generated by paratill was found to impact the soil's biological condition under no-tillage. Paratill promoted the development of root systems without significantly impacting the biomass of the corn. In addition, it created an edaphic environment that conditioned those processes that regulate the microbial population. Moreover, paratill stimulated the dynamics of the organic matter without modifying the fauna of the soil, which settled down during the period of implementation of the no-tillage system. The multivariate methods were sufficient to integrate the various attributes of the system.
\end{abstract}

Key words: Biological condition, no-tillage, paratill, Zea mays.

\section{Introducción}

El suelo es uno de los ecosistemas más complejos y diversos que existen en la naturaleza. Es un ambiente que combina las fases sólida, líquida y gaseosa formando una matriz tridimensional. La compleja naturaleza físico-química, su estructura porosa y el contenido de materia orgánica en diversas fases de descomposición y complejidad, proporcionan una heterogeneidad trófica y de hábitat que permite en él la coexistencia de una gran diversidad de organismos (Zerbino y Altier, 2006). El ciclo

Recibido 19 noviembre 2007. Aceptado 06 abril 2008.

1'Dirigir correspondencia a C. Alzugaray: calzugar@unr.edu.ar de los nutrientes, junto con la estructura edáfica y otras propiedades del suelo, son sustancialmente reguladas por la actividad de una comunidad de microorganismos y diversas especies de invertebrados (Lavelle, 1997).

El uso del suelo con fines productivos genera alteraciones en la interrelación de las propiedades edáficas. En las últimas décadas en la región pampeana de Argentina se produjo un cambio importante del uso del suelo. Este cambio estuvo asociado con el incremento del área cultivada con soja, lo que alteró las condiciones biofísicas de los suelos de la región pampeana.

Con la introducción de cultivares de soja 
resistentes a glifosato ha predominado la siembra directa, manteniendo el suelo sin laboreo (Vilche et al., 2006). Este sistema de labranza mantiene la cubierta vegetal del suelo y ha permitido mejorar la productividad y reducir la erosión hídrica en la región pampeana. No obstante, suele compactar el suelo en forma considerable debido a la consolidación natural de las partículas y al tráfico de maquinarias (Domínguez et al., 2000; Marelli y Arce, 1989).

La compactación afecta significativamente la interrelación entre las propiedades físicas, químicas y biológicas del suelo. Incluso incide sobre los rendimientos de los cultivos (Bonel et al., 2005; Abu Hamdeh, 2003). Esta restricción mecánica se puede solucionar con una labor profunda, capaz de soltar mecánicamente el suelo. Así es posible preservar la cobertura vegetal, minimizar los efectos negativos sobre los agentes formadores de biocanales y lograr una mayor exploración radical (Martino, 1998). La condición biológica del suelo es muy sensible a los disturbios ocasionados por el laboreo. Se considera que la biomasa microbiana es un excelente indicador de las variaciones en los sistemas de laboreo y rotaciones de cultivos (Alvarez, 2000; Karlen et al., 1997).

Por otra parte, la respiración basal es un parámetro que monitorea la actividad de la biomasa y la mineralización de la materia orgánica (Tate, 1995; Anderson y Domsch, 1978), detectando las modificaciones en el ecosistema (Paul et al., 1999). El nivel de respiración depende de la humedad y temperatura edáfica que a su vez dependen del manejo cultural del suelo, incidiendo también la fauna del mismo (Singh y Gupta, 1977). La macrofauna, incluso, responde de modo diferencial de acuerdo a los taxones (Altier $e t$ al., 2005; Alvarez Castillo, 2005).

En general, se analizan los efectos de las labranzas sobre las propiedades del suelo, considerándolas separadamente mediante métodos univariados. Sin embargo, es preferible utilizar procedimientos estadísticos multivariados, si se considera el suelo como una unidad natural en un continuo tridimensional dinámico y se quiere conocer las interrelaciones entre varios atributos del mismo (Nolin et al., 1989). Diversos autores han recurrido a esta metodología para evaluar simultáneamente múltiples características como las propiedades del suelo y del manejo (Vilche, 2005; Ferraro, 2005; Vilche et al., 2002; Pires Da Silva et al., 2001). Cuando se trabaja en agroecosistemas, esta visión es clave debido a su complejidad y al gran número de interacciones que se establecen en ellos (Karlen et al., 2003).

Los objetivos de este trabajo fueron: i. Evaluar los cambios biológicos de un suelo en un sistema de siembra directa escarificada bajo cultivo de maíz, a través de la actividad microbiológica, meso y macrofauna, sistema de raíces del maíz, biomasa acumulada del cultivo y nutrientes vinculados a la materia orgánica del suelo; ii. Probar el análisis multivariado como herramienta de evaluación para el conjunto de variables elegidas.

\section{Materiales y métodos}

\section{Localidad}

Este estudio se realizó en el Campo Experimental Villarino, Zavalla, Provincia de Santa Fe $\left(33^{\circ} 01^{\prime}\right.$ S, $60^{\circ} 53^{\prime \prime}$ O). La región se caracteriza por tener un clima húmedo a subhúmedo mesotermal. El suelo es un Argiudol vértico, profundo, moderadamente bien drenado, desarrollado sobre sedimento loéssico, con un horizonte superficial franco limoso de $20 \mathrm{~cm}$ de profundidad, con mezcla de escasos agregados esféricos medianos y bloques sub-angulares. Presenta un horizonte BA franco arcillo limoso con bloques sub-angulares y angulares, seguido por un horizonte Bt de textura arcillosa, con estructura prismática moderada a fuerte que se inicia a los $40 \mathrm{~cm}$ y se extiende hasta los $140 \mathrm{~cm}$. Este horizonte presenta planos de deslizamiento y abundantes revestimientos de arcilla en los prismas. El horizonte $\mathrm{C}$ es un horizonte suelto que se desarrolla desde los $140 \mathrm{~cm}$.

\section{Cultivo y labores del suelo}

Este estudio se realizó en un lote sembrado directamente por 14 años, donde se cultivó sucesivamente alfalfa para corte, maíz, soja, trigo en combinación con soja de modo que hubo 
una relación 3:9 años, entre la fase ganadera y la fase agrícola.

La siembra directa escarificada (SDE) consistió en una labor de escarificado en agosto de 2005 con un equipo tipo paratill (Dolbi, Reconquista, Argentina), provisto de seis púas rígidas y rectas separadas a $35 \mathrm{~cm}$. Se obtuvo una profundidad efectiva de trabajo entre 27 y 30 $\mathrm{cm}$. La humedad del suelo al efectuar la labor fue de $19 \%(\mathrm{p} / \mathrm{p})$ en el estrato de 0-30 cm. A los $45 \mathrm{~d}$ de realizado el escarificado se sembró 25,8 $\mathrm{kg} \cdot \mathrm{ha}^{-1}$ de maíz, que se fertilizó con $60 \mathrm{~kg} \cdot \mathrm{ha}^{-1}$ de fosfato diamónico más $80 \mathrm{~kg} \cdot \mathrm{ha}^{-1}$ de urea.

A la siembra y a la cosecha del maíz se extrajeron diez sub-muestras a 0-5, 5-15 y $15-30 \mathrm{~cm}$ de profundidad desde las parcelas bajo siembra directa (SD) y SDE. En cada muestra de suelo, se determinó el carbono orgánico total (Nelson y Sommers, 1982), sulfatos por turbidimetría (Tabatabai, 1982), nitratos (Harper modificado, Jackson, 1964), fosfatos (Bray y Kurtz, 1945) ( $\mathrm{n}=12$ por tratamiento). Además, se determinó el carbono de la biomasa microbiana (Jenkinson y Powlson, 1976) y la actividad respiratoria microbiana en las profundidades $0-10$ y $10-30$ $\mathrm{cm}(\mathrm{n}=12$ por tratamiento) (Frioni, 2006).

Se excavaron 10 pozos de 0,4 x $1,0 \mathrm{~m}$ donde se determinó la profundidad alcanzada por las raíces en el estadio de quinta hoja desarrollada (V5) del maíz. A la cosecha se evaluó el peso seco aéreo, del grano y de las raíces en 20 plantas por tratamiento. El sistema radical se extrajo con pala considerando la profundidad de labor $(0-30 \mathrm{~cm})$. Se determinó el peso seco $\left(75^{\circ} \mathrm{C}\right.$ hasta peso estable) del material aéreo (PSA), excluyendo el grano, y el peso seco las raíces (PSR) El peso del grano (PSG) se determinó con $13,5 \%$ de humedad. Se calculó la relación entre el peso seco radical y el peso seco aéreo, excluyendo el grano (PSR/PSA).

Por otra parte, a la cosecha se muestreó el suelo al azar con barrenos de $15 \mathrm{~cm}$ de diámetro por $30 \mathrm{~cm}$ de profundidad, obteniendo aproximadamente $5300 \mathrm{~cm}^{3}$ de suelo para cada muestra ( $\mathrm{n}=12$ por tratamiento). La humedad edáfica en el momento del muestreo fue de $22 \%$ (p/p). En cada muestra se evaluó: 1. Diversidad de la macro y mesofauna $(\mathrm{H}=$
$\Sigma$ Pi $\mathrm{x} \ln (\mathrm{Pi})$; donde $\mathrm{Pi}=$ proporción del taxón en la muestra), 2. Número de taxones presentes, 3. Número de individuos por taxón, 4. Actividad de la fauna (cantidad de nidos, madrigueras, restos de mudas, presencia de excretas), 5. Número de galerías de la fauna, 6 . Abundancia y características de las raíces del año vivas y galerías de raíces de cultivos antecesores, 7. Residuos orgánicos en descomposición y 8. Presencia de micelios de hongos. La macro y mesofauna se determinó por recuento de animales visibles, directamente los que se discriminaron por Phyla, Clases y Ordenes taxonómicos. Además, se contaron las veces en que se encontraron krotovinas al realizar el análisis de cada bloque de suelo.

\section{Diseño y análisis estadísticos}

El diseño experimental fue en bloques completos aleatorizados con cuatro repeticiones, siendo los tratamientos siembra directa (SD) y siembra directa escarificada (SDE). La unidad experimental estuvo constituida por parcelas de $210 \mathrm{~m}^{2}$.

Primeramente se compararon los tratamientos por análisis estadísticos univariados. Para los parámetros edáficos y de materia seca del maíz se realizó una prueba de diferencias apareadas (Giubileo et al., 2006). Para los parámetros microbiológicos se realizó análisis de varianza y los promedios se separaron de acuerdo con la comparación múltiple de Duncan (InfoStat, Facultad de Ciencias Agrarias, Universidad Nacional de Córdoba, Argentina, 2004). Previo a los análisis estadísticos, las variables (x) peso seco de granos, raíces y parte aérea se normalizaron como logaritmo de x (Shapiro y Wilk, 1965). Para los siguientes parámetros relacionados con la fauna del suelo, número de individuos y taxones, actividad y galerías y residuos orgánicos en descomposición, micelio de hongos, galería de raíces y krotovinas, se utilizó la prueba de Wilcoxon para datos no paramétricos (Hollander y Wolfe, 1973).

En una segunda etapa, los datos se analizaron con técnicas estadísticas multivariadas de ordenamiento y clasificación, empleando las siguientes escalas de evaluación:

a. Parámetros edáficos y microbiológicos: 
Carbono orgánico, $1<2570$ y $2>2570 \mathrm{~kg} \cdot \mathrm{ha}^{-1}$. Nitratos: $1<0,01 ; 2=0,015$ a 0,035 y $3>0,035$ $\mathrm{kg} \cdot \mathrm{ha}^{-1}$. Sulfatos: $1<0,002 ; 2=0,002$ a 0,004 ; $3=0,0041$ a 0,008 y $4>0,008 \mathrm{~kg} \cdot \mathrm{ha}^{-1}$. Fosfatos: $1<0,07$ y $2>0,07 \mathrm{~kg} \cdot \mathrm{ha}^{-1}$. Biomasa microbiana: $1<10$ y $2>10 \mathrm{mg}$ de $\mathrm{C}$ por $100 \mathrm{~g}$ de suelo seco. Respiración: $\quad 1<10,2=10-20,3=20-30$ y 4 $>30 \mathrm{mg} \mathrm{CO} 2$ por $100 \mathrm{~g}$ de suelo seco en $10 \mathrm{~d}$.

b. Variables referentes al cultivo. PSR/ PSA: $1=0,10$ a 0,$30 ; 2=0,31$ a 0,60 y $3=0,61$ a 1 . Profundidad de las raíces $(0-30 \mathrm{~cm})$ en el estado V5 del desarrollo fenológico del maíz fue $1=$ hasta $27 \mathrm{~cm}$ y $2>27 \mathrm{~cm}$.

c. Aspectos biogénicos asociados con la fauna de suelo. Actividad y galerías de la fauna: $1=$ una galería y por lo menos tres sitios con signos de actividad, 2 = dos galerías y cuatro sitios, 3 $=$ tres galerías y cinco sitios. Galerías creadas por raíces de cultivos precedentes y la presencia de raíces del año: 1 = una a cinco galerías y escasas raíces vivas, $2=$ seis a diez galerías y mayor cantidad de raíces, $3>$ diez galerías y profuso desarrollo radical. Presencia de fauna: $1=1$ a $3,2=4$ a $6,3=7$ a 9 y $4>10$ individuos. Diversidad de fauna: $1=1$ a 3 taxones, $2=$ 4 a 6 taxones y $3=7$ a 10 taxones. Residuos orgánicos en descomposición en el suelo: $1=$ observado en un sitio, $2=2$ a 4 sitios y $3>4$ sitios. Presencia de hongos: $1=$ micelio visible en 5 sitios y $2=$ en más de 5 sitios y dentro de galerías. Presencia de krotovinas: $1<2$ y $2>2$. Se construyó un matriz de muestras $\mathrm{x}$ variables y los datos se procesaron empleando PC-ORD (Version 4, Sofware Design, Oregon. EUA, 1999). Se realizó un ordenamiento por el método de componentes principales utilizando una matriz de varianza y covarianza. Los componentes principales que explicaron más del 5\% de la varianza total fueron considerados significativos (Wander y Bolero, 1999). Para la clasificación de los datos se utilizó el método de Ward (1963).

\section{Resultados}

A los 45 d de realizada la escarificación del suelo, sólo el contenido de sulfatos mostró diferencias significativas $(\mathrm{p}<0,05)$ a favor de la SDE. Con la excepción de los sulfatos, los contenidos de nitratos $\mathrm{y}$ fosfatos fueron significativamente $(\mathrm{p}$ $<0,05$ ) mayores en el suelo SDE que en SD, luego de 10 meses de la escarificación del suelo (Cuadro 1).

Hubo una interacción significativa $(\mathrm{p}<0,05)$ entre tratamientos (SD y SDE) y profundidad de muestreo del suelo. Independientemente de la profundidad de muestreo del suelo, tanto la biomasa microbiana como la respirometría fueron significativamente $(\mathrm{p}<0,05)$ superiores en el suelo escarificado respecto de la siembra directa, a los 45 d de realizada la escarificación. Los valores disminuyeron hacia el fin de ciclo del cultivo de maíz, luego de 10 meses de

Cuadro 1. Parámetros edáficos determinados en un suelo con siembra directa y siembra directa escarificada

Table 1. Soil parameters assessed in a soil under no-tillage and no-tillage with soil scarification.

\begin{tabular}{|c|c|c|c|c|}
\hline \multirow[b]{2}{*}{ Manejo del suelo } & \multicolumn{4}{|c|}{ Parámetros edáficos } \\
\hline & $\begin{array}{l}\text { Nitratos } \\
\mathrm{mg} \cdot \mathrm{ha}^{-1}\end{array}$ & $\begin{array}{l}\text { Sulfatos } \\
\mathrm{mg} \cdot \mathrm{ha}^{-1}\end{array}$ & $\begin{array}{l}\text { Fosfatos } \\
\mathrm{mg} \cdot \mathrm{ha}^{-1}\end{array}$ & $\begin{array}{c}\text { Carbono orgánico total } \\
\qquad \cdot \mathrm{ha}^{-1}\end{array}$ \\
\hline \multicolumn{5}{|c|}{45 d después de la escarificación con paratill } \\
\hline Siembra directa & $7,0 \mathrm{a}^{2}$ & $1,0 \mathrm{~b}^{2}$ & $7,7 \mathrm{a}^{2}$ & \\
\hline Siembra directa en suelo escarificado ${ }^{1}$ & 8,0 a & $3,0 \mathrm{a}$ & $7,7 \mathrm{a}$ & \\
\hline
\end{tabular}

10 meses después de la escarificación con paratill

\begin{tabular}{|c|c|c|c|}
\hline Siembra directa & $50,0 \mathrm{a}^{2}$ & $7,0 \mathrm{a}^{2}$ & $68,0 \mathrm{~b}^{2}$ \\
\hline Siembra directa en suelo escarificado ${ }^{1}$ & $37,0 \mathrm{~b}$ & $5,0 \mathrm{~b}$ & 86,0 a \\
\hline
\end{tabular}

${ }^{1}$ Escarificación realizada con un equipo tipo paratill (Dolbi, Reconquista, Argentina), provisto de seis púas rígidas y rectas separadas a 35 cm. ${ }^{2}$ Promedios seguidos por igual letra en cada columna no son estadísticamente diferentes entre si según la prueba de diferencias apareadas $(\mathrm{p}<0,05)$.

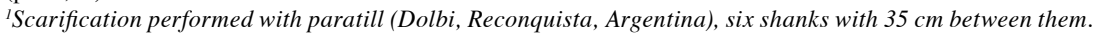

${ }^{2}$ Means followed by the same letter in each column, are not statistically different by paired differences test $(p<0.05)$. 
Cuadro 2. Parámetros microbiológicos determinados en un suelo con siembra directa y con siembra directa escarificada. Table 2. Soil microbiological parameters assessed in a soil under no-tillage and no-tillage with scarification.

\begin{tabular}{|c|c|c|c|c|c|c|c|c|}
\hline \multirow{3}{*}{$\begin{array}{l}\text { Suelo } \\
\text { Estratos } \\
\mathrm{cm}\end{array}$} & \multicolumn{8}{|c|}{ Parámetros microbiológicos por $100 \mathrm{~g}$ de suelo $\operatorname{seco}^{1}$} \\
\hline & \multicolumn{4}{|c|}{$\begin{array}{l}45 \text { días después de la } \\
\text { escarificación del suelo }\end{array}$} & \multicolumn{4}{|c|}{$\begin{array}{l}10 \text { meses después de la } \\
\text { escarificación del suelo }\end{array}$} \\
\hline & \multicolumn{2}{|c|}{$\begin{array}{c}\text { Biomasa } \\
\mathrm{C}, \mathrm{mg}\end{array}$} & \multicolumn{2}{|c|}{$\begin{array}{l}\text { Respirometría } \\
\mathrm{CO}_{2}, \mathrm{mg}\end{array}$} & \multicolumn{2}{|c|}{$\begin{array}{c}\text { Biomasa } \\
\mathrm{C}, \mathrm{mg}\end{array}$} & \multicolumn{2}{|c|}{$\begin{array}{l}\text { Respirometría } \\
\mathrm{CO}_{2}, \mathrm{mg}\end{array}$} \\
\hline \multicolumn{9}{|l|}{ Siembra directa ${ }^{2}$ : } \\
\hline $0-10$ & \multicolumn{2}{|c|}{$19,7 c^{3}$} & \multicolumn{2}{|c|}{$20,0 \mathrm{c}^{3}$} & \multicolumn{2}{|c|}{$16,0 \mathrm{a}^{3}$} & \multicolumn{2}{|c|}{$15,7 \mathrm{a}^{3}$} \\
\hline $10-30$ & \multicolumn{2}{|c|}{$21,2 \mathrm{c}$} & \multicolumn{2}{|c|}{$27,7 \mathrm{~b}$} & \multicolumn{2}{|c|}{$9,3 \mathrm{c}$} & \multicolumn{2}{|c|}{$12,0 \mathrm{~b}$} \\
\hline \multicolumn{9}{|c|}{ Siembra directa en suelo escarificado ${ }^{2}$ : } \\
\hline $0-10$ & \multicolumn{2}{|c|}{$25,0 \mathrm{~b}^{3}$} & \multicolumn{2}{|c|}{$36,0 \mathrm{a}^{3}$} & \multicolumn{2}{|c|}{$14,0 \mathrm{~b}^{3}$} & \multicolumn{2}{|c|}{$13,4 b^{3}$} \\
\hline $10-30$ & \multicolumn{2}{|c|}{$31,0 \mathrm{a}$} & \multicolumn{2}{|c|}{$37,3 \mathrm{a}$} & \multicolumn{2}{|c|}{$9,7 \mathrm{c}$} & \multicolumn{2}{|c|}{$13,7 \mathrm{~b}$} \\
\hline Análisis de varianza & & & & & & & & \\
\hline $\begin{array}{l}\text { Causa de } \\
\text { variación }\end{array}$ & $\mathrm{F}$ & $\mathrm{p}$ & $\mathrm{F}$ & $\mathrm{p}$ & $\mathrm{F}$ & $\mathrm{p}$ & $\mathrm{F}$ & $\mathrm{p}$ \\
\hline Tratamiento $(\mathrm{T})$ & 0,68 & 0,42 & 8,86 & 0,08 & 9,36 & 0,006 & 0,11 & 0,74 \\
\hline Profundidad de muestreo $(\mathrm{P})$ & 0,84 & 0,37 & 0,52 & 0,48 & 26,81 & 0,0001 & 30,26 & 0,0001 \\
\hline $\mathrm{T} \times \mathrm{P}$ & 0,06 & 0,8 & 0,19 & 0,67 & 5,30 & 0,03 & 8,85 & 0,007 \\
\hline
\end{tabular}

${ }^{1}$ Suelo seco a $75^{\circ} \mathrm{C}$ hasta peso estable.

${ }^{2}$ Escarificación del suelo realizada con un equipo tipo paratill (Dolbi, Reconquista, Argentina), provisto de seis púas rígidas y rectas separadas a $35 \mathrm{~cm}$.

${ }^{3}$ Promedios seguidos por igual letra en cada columna no son estadísticamente diferentes entre si según la prueba de comparación múltiple de Duncan $(\mathrm{p}<0,05)$.

${ }^{I}$ Soil dried at $75^{\circ} \mathrm{C}$ to a constant weight.

${ }^{2}$ Soil scarification performed with paratill (Dolbi, Reconquista, Argentina), six shanks with $35 \mathrm{~cm}$ between them.

${ }^{3}$ Means followed by the same letter in each column are not statistically different according to Duncan's multiple range test $(p<0.05)$.

escarificar el suelo y hubo sólo diferencias significativas en los primeros $10 \mathrm{~cm}$ de suelo a favor de SD (Cuadro 2).

La profundidad radical en el estadio V5 del maíz fue significativamente $(\mathrm{p}<0,05)$ mayor en SDE que en SD con promedios de profundización radical de 31,2 y $26,2 \mathrm{~cm}$, respectivamente. El PSG fue 167,30 y 173,8 g.mazorca ${ }^{-1}$ en SD y SDE, respectivamente sin mostrar diferencias estadísticamente significativas. Del mismo modo las diferencias en el PSA no fueron significativas con 130,1 y 152,8 g.planta ${ }^{-1}$ en $\mathrm{SD}$ y SDE, respectivamente. La proporción en granos del peso seco total aéreo fue de $47,2 \%$ para SD y $44,7 \%$ para SDE, no significativa. El PSR alcanzado por el sistema radical a la cosecha en la profundidad $0-30 \mathrm{~cm}$ fue significativamente superior $(\mathrm{p}<0,05)$ en SDE (69,2 y 37,3 g SDE y SD respectivamente). Los valores promedio de PSR/PSA fueron de $0,29 \mathrm{y}$ 0,45 en SD y SDE $(p<0,05)$, respectivamente. La SD no se diferenció significativamente de la
SDE en los aspectos relacionados con la meso y macrofauna (Cuadro 3). El índice de diversidad promedio de las muestras de SD fue de $0,76 \mathrm{y}$ de 0,71 en las muestras de SDE, sin diferencias significativas entre tratamientos.

La actividad de la fauna y sus galerías fue significativamente $(\mathrm{p}<0,05)$ mayor en SD que en SDE. En cambio, la presencia de galerías de raíces de cultivos antecesores, residuos orgánicos en descomposición, presencia de micelio de hongos y krotovinas fueron estadísticamente similares (Cuadro 4).

En el análisis de componentes principales realizado con el conjunto de todas las variables se consideraron significativos los seis primeros ejes que explicaron el $85,52 \%$ de la variancia acumulada (Cuadro 5, Figura 1). Se destaca que los tres primeros ejes del ordenamiento sumaron el $66,03 \%$ de la varianza total. El primer componente principal (CP1) explicó el 34,13\% de la varianza y separó SD de SDE. De este 
Cuadro 3. Densidad promedio de la meso y macrofauna por taxones determinados en un suelo con siembra directa y siembra directa escarificada.

Table 3. Mean density of meso- and macrofauna by taxa in a soil under no-tillage and no-tillage with scarification.

\begin{tabular}{|c|c|c|c|c|}
\hline \multirow[t]{2}{*}{ Taxón } & \multicolumn{2}{|c|}{ Siembra directa ${ }^{1}$} & \multicolumn{2}{|c|}{ Siembra directa escarificada } \\
\hline & $\begin{array}{c}\text { Densidad } \\
\text { no. } \cdot \mathrm{m}^{-2}\end{array}$ & $\begin{array}{c}\mathrm{CV}^{2} \\
\%\end{array}$ & $\begin{array}{c}\text { Densidad } \\
\text { no. } \cdot \mathrm{m}^{-2}\end{array}$ & $\begin{array}{c}\mathrm{CV}^{2} \\
\%\end{array}$ \\
\hline \multicolumn{5}{|c|}{ Phylum Anelida, Clase Polychaeta: } \\
\hline & 46,6 & 1,4 & 23,3 & 1,0 \\
\hline \multicolumn{5}{|c|}{ Phylum Arthropoda, Clase Aracnida: } \\
\hline & 5,8 & 3,0 & 5,8 & 3,0 \\
\hline \multicolumn{5}{|c|}{ Phylum Arthropoda, Clase Chilopoda, Diplopoda: } \\
\hline & 11,7 & 2,0 & 29,1 & 1,7 \\
\hline \multicolumn{5}{|c|}{ Phylum Arthropoda, Clase Hexapoda: } \\
\hline Colembola & 116,5 & 1,4 & 52,4 & 1,0 \\
\hline \multicolumn{5}{|c|}{ Phylum Arthropoda, Clase Insecta: } \\
\hline Hymenoptera & 81,6 & 1,9 & 46,6 & 2,0 \\
\hline \multicolumn{5}{|l|}{ Coleoptera } \\
\hline Adultos & 23,3 & 1,3 & 23,3 & 1,0 \\
\hline Larvas & 23,3 & 1,1 & 29,1 & 2,0 \\
\hline Orthoptera & 0,0 & 0,0 & 5,8 & 3,0 \\
\hline Thysanoptera & 5,8 & 3,0 & 0,0 & 0,0 \\
\hline Blattaria & 0,0 & 0,0 & 5,8 & 3,0 \\
\hline
\end{tabular}

${ }^{1}$ Diferencias entre promedios estadísticamente no significativas según la prueba de Wilcoxon $(\mathrm{p}<0,05)$.

${ }^{2} \mathrm{CV}=$ coeficiente de variación.

${ }^{\prime}$ Means are not statistically different by Wilcoxon's test $(p<0.05)$.

${ }^{2} \mathrm{CV}=$ coefficient of variation.

modo la SDE se ubicó a la derecha del gráfico en función de la mayor profundidad radical alcanzada en V5, la mayor disponibilidad de nitratos y sulfatos en los dos momentos de muestreo y la menor cantidad de fosfatos a la cosecha. Las muestras de SD, localizadas a la izquierda se caracterizaron por presentar más actividad biogénica provocada por la fauna, numerosa cantidad de galerías generadas por las raíces de cultivos antecesores, con mayor presencia de krotovinas. Respecto de los nutrientes disponibles SD presentó menor cantidad a la siembra y mayor a la cosecha que SDE. El CP2 explicó un 21,3\% de la varianza considerando número de individuos, diversidad de la fauna y galerías de raíces de cultivos antecesores. Si bien estas variables no discriminan claramente entre tipos de labranzas (SD y SDE), se pueden distinguir dos grupos dentro del sistema de SD. Así en el cuadrante inferior izquierdo del ordenamiento se observa el grupo con mayor densidad y diversidad de fauna. En el CP3 se destacaron los parámetros microbiológicos, respirometría y biomasa, en los dos momentos de muestreo. En los tres ejes subsiguientes la relación PSR/PSA y los residuos orgánicos en descomposición fueron las variables de mayor peso.

Con el método de Ward no se logró diferenciar los cambios generados entre SD y SDE cuando se utilizó el conjunto completo de variables. Pero al considerar separadamente dos grupos de variables, el primer dendrograma con los parámetros edáficos y microbiológicos (Figura 2A) pone de manifiesto la importancia de estas variables para caracterizar el ambiente edáfico ya que diferencia notoriamente las muestras provenientes de $\mathrm{SD}$ y SDE. El segundo dendrograma (Figura 2B) agrupó el $75 \%$ de las muestras de SDE en el extremo de menor diversidad de meso y macrofauna y el resto se desplegó en un gradiente de diversidad creciente, donde predominan las muestras proveniente de SD. 
Cuadro 4. Efecto de la siembra directa y de la siembra directa escarificada sobre los aspectos biológicos del suelo. Table 4. Effect of no-tillage and no-tillage with scarification on soil biological properties.

\begin{tabular}{|c|c|c|c|c|}
\hline \multirow[t]{2}{*}{ Parámetros } & \multicolumn{2}{|c|}{ Siembra directa } & \multicolumn{2}{|c|}{ Siembra directa escarificada } \\
\hline & no. $m^{-2}$ & $\mathrm{CV}^{2}, \%$ & no. $\cdot m^{-2}$ & $\mathrm{CV}^{2}, \%$ \\
\hline Galerías de la fauna (GF) & $492,4 a^{1}$ & 0,7 & $152,8 b^{1}$ & 0,9 \\
\hline Sitios con actividad de la fauna (AF) & $152,8 \mathrm{a}$ & 0,2 & $56,6 \mathrm{~b}$ & 1,1 \\
\hline $\begin{array}{l}\text { Individuos totales de meso y } \\
\text { macrofauna (MMF) }\end{array}$ & $314,7 \mathrm{a}$ & 1,0 & $221,5 \mathrm{a}$ & 0,7 \\
\hline $\begin{array}{l}\text { Galerías creadas por raíces de } \\
\text { cultivos antecesores (GR) }\end{array}$ & $571,6 \mathrm{a}$ & 1,3 & $520,7 \mathrm{a}$ & 1,2 \\
\hline $\begin{array}{l}\text { Sitios con presencia de residuos } \\
\text { orgánicos en descomposición (MO) }\end{array}$ & $62,2 \mathrm{a}$ & 0,2 & $67,9 \mathrm{a}$ & 0,4 \\
\hline $\begin{array}{l}\text { Sitios con presencia de micelio de } \\
\text { hongos }(\mathrm{MH})\end{array}$ & $34,0 \mathrm{a}$ & 1,1 & $45,2 \mathrm{a}$ & 1,4 \\
\hline Sitios con presencia de krotovinas $(\mathrm{K})$ & $22,6 a$ & 1,3 & $11,3 \mathrm{a}$ & 1,4 \\
\hline
\end{tabular}

${ }^{1}$ Promedios seguidos por igual letra en cada fila no son estadísticamente diferentes entre si según la prueba de Wilcoxon $(\mathrm{p}<0,05)$ ${ }^{2} \mathrm{CV}=$ coeficiente de variación.

${ }^{\prime}$ Means followed by the same letter in each row are not statistically different by Wilcoxon's test $(p<0.05)$.

${ }^{2} \mathrm{CV}=$ coefficient of variation.

\section{Discusión}

A los $45 \mathrm{~d}$ de la escarificación hubo un incremento significativo de sulfatos en SDE respecto de $\mathrm{SD}$, pero no hubo diferencias estadísticamente significativas en el contenido de nitratos y fosfatos disponibles en el suelo bajo SD. Si bien la disponibilidad de fosfatos aumentó después de 10 meses de escarificado

Cuadro 5. Análisis de componentes principales de las variables edáficas, microbiológicas y otros aspectos vinculados a actividad biológica del suelo.

Table 5. Principal component analysis of soil, microbiology variables and other aspect related to biological the biological activity of the soil.

\begin{tabular}{lccc}
\hline Componente & Autovector $^{1}$ & $\begin{array}{c}\text { Varianza } \\
\text { principal } \\
\%\end{array}$ & $\begin{array}{c}\text { Varianza } \\
\text { acumulada } \\
\%\end{array}$ \\
\hline CP1 & 54,23 & 34,13 & 34,13 \\
CP2 & 33,89 & 21,33 & 55,46 \\
CP3 & 16,78 & 10,57 & 66,03 \\
CP4 & 12,05 & 7,58 & 73,61 \\
CP5 & 10,53 & 6,63 & 80,24 \\
CP6 & 8,39 & 5,28 & 85,52 \\
\hline
\end{tabular}

${ }^{1}$ Cada componente principal (CP1 a CP6) se denomina autovector (= vector eigen) y explica la varianza que absorbe.

${ }^{\prime}$ Each principal component (PC to PC6) is named autovector (= eigen vector) and explains the associated variance.

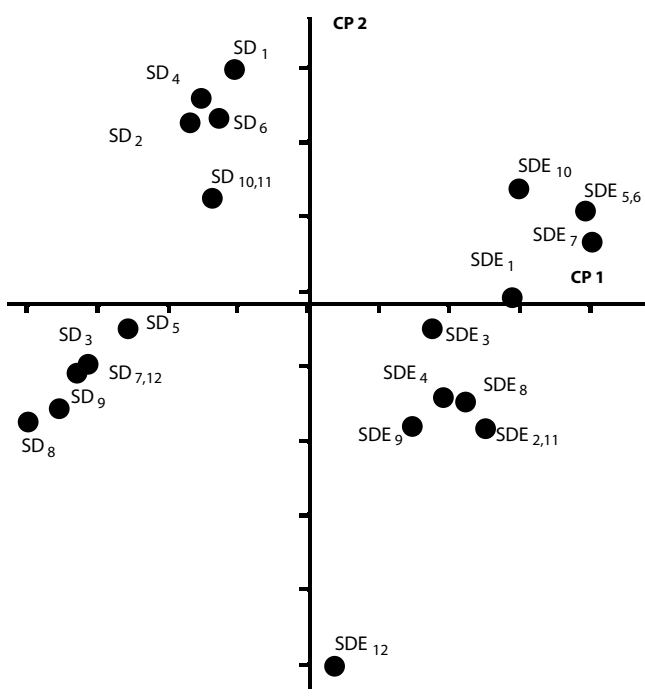

Figura 1. Diagrama de la distribución de las muestras en el plano formado por los dos primeros componentes principales (CP1 y CP2), considerando las variables edáficas, microbiológicas y aspectos vinculados a la actividad biológica del suelo. $\mathrm{SD}=$ siembra directa, $\mathrm{SDE}=$ siembra directa escarificada (los números corresponden a diferentes sitios de muestreo).

Figure 1. Scatter diagram of the samples obtained by the two first principal components (PC1 and PC2), considering the edaphic, microbiology variables and features related to biological activity. $S D=$ No-tillage samples and NTP = no-tillage with paratill (numbers belong to different samples collected sites). 


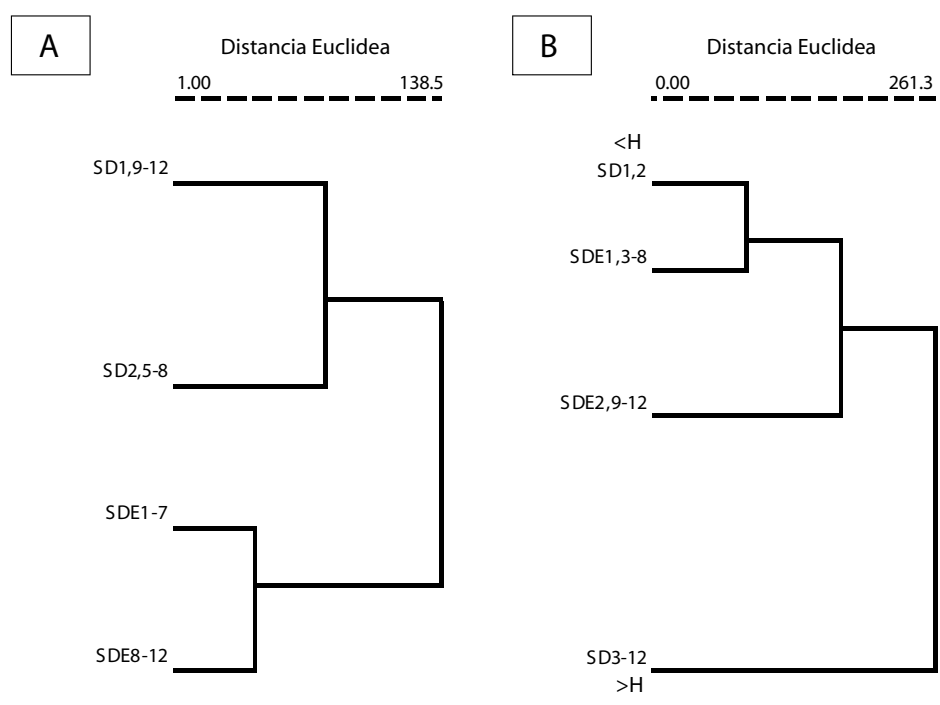

Figura 2. Clasificación obtenida por el método de Ward en siembra directa y siembra directa escarificada. A. Dendrograma de los parámetros edáficos: carbono orgánico, nitratos, sulfatos y fósforo disponibles, y microbiológicos: respirometría y biomasa microbiana. B. Dendrograma de la densidad de individuos por taxón y la diversidad (H) de la meso y macrofauna. $\mathrm{SD}=$ siembra directa y $\mathrm{SDE}=$ siembra directa escarificada con paratill (los números corresponden a diferentes sitios de muestreo).

Figure 2. Clustering by Ward's method in no-tillage and no-tillage with paratill. A. Dendrogram of soil parameters: organic carbon, nitrates, sulphates and phosphorous available and microbial biomass and microbial respiration. $B$. Dendrogram of density of individuals by taxon and diversity of macro- and mesofauna. $H=$ diversity, $S D=$ no-tillage and $S D E=$ no-tillage using paratill (numbers belong to different sampling sites).

el suelo, los sulfatos y nitratos disminuyeron. Probablemente, estas diferencias se deban a diferencias entre los ciclos que describen el comportamiento de estos nutrientes en el suelo y su relación con la materia orgánica. Los fosfatos son poco móviles, mientras que los sulfatos y nitratos son móviles, presentan una dinámica similar y muy activa en el suelo. Por lo tanto, es posible que la lixiviación y el consumo realizado por el maíz expliquen la disminución obtenida.

El comportamiento de las raíces sugiere, que como consecuencia del aflojamiento provocado por la labor de escarificado, se produjo una mayor exploración del suelo en SDE que en SD, similar a lo observado previamente para maíz (Martino, 1998). Esto pudo haber originado un mayor consumo de nutrientes, particularmente de nitratos (Braim et al., 1984; Hipps y Hodgson, 1988). De acuerdo con Ide et al. (1984) la labor profunda aumentaría la absorción de fosfatos, posiblemente por hacerlo más accesible como resultado de una menor compactación del suelo. Además, podría haber facilitado la descomposición de los compuestos orgánicos fosfatados, incrementando la fracción disponible.

En gran medida las reacciones que tienen lugar en los ciclos de nutrientes, son posibles gracias a la actividad microbiana del suelo, este es el caso de los fosfatos, nitratos y sulfatos (Marasas, 2002). Los valores de respirometría obtenidos en SDE fueron considerablemente superiores a los de SD, probablemente por una mejor oxigenación del suelo y mayor disponibilidad de compuestos orgánicos para los microorganismos. Es importante considerar que inicialmente, la biomasa obtenida en $\mathrm{SDE}$ fue mayor que en $\mathrm{SD}$, lo que sugiere condiciones favorables para el desarrollo de los microorganismos. Estos resultados coinciden con lo informado previamente para un suelo subsolado bajo siembra directa (Torella et al., 2004). No obstante, a los 10 
meses de escarificado el suelo, los parámetros microbiológicos tendieron a uniformizarse. $\mathrm{La}$ similitud de la actividad respiratoria SD y SDE luego de 10 meses de la escarificación del suelo estaría indicando la escasa perdurabilidad del efecto de la escarificación.

Aun cuando en este trabajo no se determinó la humedad y temperatura del perfil del suelo, datos obtenidos en maíz colindante, en similares condiciones experimentales, en SDE tuvieron humedades entre 25 y $40 \%$ por debajo las humedades registradas en SD a 0-40 $\mathrm{cm}$ de profundidad durante el ciclo de cultivo (S. Montico, com. pers.). Considerando que la actividad microbiana decrece al disminuir el contenido hídrico del suelo, es posible que la disminución de la actividad biológica y de la biomasa en SDE se deba a una menor humedad del suelo en el espesor afectado por el escarificador (Orchard and Cook, 1983; Sylvia et al., 1999). Por otra parte, la labranza profunda puede incrementar la evapotranspiración, ya que favorece la exploración radical lo que aumenta el consumo de agua, disminuyendo el contenido hídrico del suelo (Martino, 1998).

En la siembra directa se presenta una importante estratificación de la materia orgánica (Domínguez et al., 2004). Sin embargo, la fragmentación generada por el escarificador probablemente incrementó la disponibilidad de oxígeno y la mineralización del C orgánico, coincidiendo el efecto producido por el uso de paraplow (Hipp y Hodgson (1988). Esta fragmentación del suelo, pudo favorecer la acción de los microorganismos sobre los compuestos químicos de menor estabilidad química. Es conocido que el aumento en la porosidad producto de este tipo de labores, se debe casi exclusivamente al efecto sobre los poros de mayor tamaño (Bonel et al., 2004; Pikul et al., 1990; Martino, 1998; Hipp y Hodgson, 1988).

Los resultados obtenidos en este trabajo confirman que los indicadores relacionados con la materia orgánica y la respirometría permiten diferenciar situaciones de manejo del suelo a corto plazo (Romaniuk et al., 2006).

Si bien la labor profunda alteró el sistema de galerías, particularmente en aquellas originadas por la fauna, no fue lo suficientemente importante como para disminuir drásticamente la actividad y el número de individuos de la fauna edáfica. La población de lombrices de tierra fue menor en SDE que en SD, pero en ambos tratamientos se mantuvo dentro de valores promedios registrados para maíz bajo SD (Kladivko, 2003). La diversidad de la meso y macrofauna obtenidos en esta investigación fueron comparables con otros trabajos en la región pampeana argentina. Del mismo modo la composición específica de la macro y meso fauna fue poco sensible a las prácticas agrícolas de la región (Ferraro, 2005; Gizzi et al., 2006).

La producción de materia seca aérea (PSA) fue mayor $(p<0,07)$ en SDE que en SD; sin embargo, no hubo diferencias significativas el rendimiento en grano entre tratamientos. Probablemente las escasas precipitaciones ocurridas durante el ciclo del cultivo hayan afectado los rendimientos de grano. Las lluvias mensuales caídas entre agosto de 2005 y marzo de 2006 inclusive, fueron respectivamente de 39,6; 41,6; 53,3; 64,8; 20,9; 110; 92,6 y 107 $\mathrm{mm}$. Estos valores fueron $30,45 \%$ inferiores al promedio histórico mensual (1973/74 a 2000/01) para Zavalla, Argentina (Sacchi et al., 2002).

Si bien, el aflojamiento del suelo produjo una mayor exploración radical en el estadio V5, lo que se tradujo en una mayor producción de materia seca aérea, la escasez de precipitaciones durante el período crítico del cultivo (diciembre a enero con $97 \mathrm{~mm}$ menos del promedio histórico) incidió en la producción de granos. En este caso la labor profunda no obtuvo un aumento de rendimiento como se ha señalado previamente (Bonel et al., 2005; Martino, 1998; Azooz et al., 1995).

La varianza explicada por el CP1 indica la importancia que tienen las variables asociadas a la dinámica de los nutrientes ligados a la materia orgánica, la mayor profundidad radical alcanzada en el estado V5 y la actividad y galerías de la fauna. De acuerdo con este análisis estas variables fueron las más importantes para la identificación de los cambios provocados por la labranza profunda. El análisis de componentes principales permitió identificar las propiedades 
físico-químicas del suelo que tuvieron el mayor efecto sobre la producción de maíz en la región pampeana argentina (Maddonni et al., 1999).

Este análisis rescató la importancia la densidad y diversidad de la fauna (Cuadro 3) y galerías de raíces de cultivos antecesores, variables que no arrojaron diferencias estadísticamente significativas en el análisis univariado. No obstante, fueron las variables que mejor se correlacionaron con el CP2 y explicaron una porción muy importante de la varianza obtenida. Los parámetros microbiológicos asociados al $\mathrm{CP} 3$, muy relevantes en el análisis univariado, aquí explican sólo el $10 \%$ de la varianza, En este aspecto los resultados obtenidos no concuerdan con resultados previos, obtenidos con esta misma metodología, donde evaluaron la condición biológica del suelo bajo siembra directa en Illinois (Wander y Bollero, 1999). Sin embargo, coinciden con trabajos donde logran describir, mediante componentes principales, el estado microbiológico del suelo como indicador de fertilidad (De Cary y Hervé, 1994). Por lo tanto, el análisis multivariado permitió destacar la importancia de los datos no paramétricos que describen la condición del ambiente edáfico y la relación de las distintas variables entre sí.

En consecuencia los resultados obtenidos en este trabajo demuestran que la descompactación originada por la labranza profunda (escarificación del suelo) mediante el uso de paratill incidió en la condición biológica del suelo bajo SDE. Esta labor promovió el desarrollo del sistema radical sin incidir significativamente en la producción de maíz. El análisis multivariado fue una herramienta adecuada de evaluación que permitió integrar diferentes atributos edáficos del sistema suelo.

\section{Resumen}

La siembra directa ofrece mayor estabilidad y diversidad biológica que otros sistemas de labranza, aunque puede generar compactación en el suelo. Esta restricción se puede resolver con una labranza profunda, capaz de aflojar el suelo pero conservando la cobertura superficial. La condición biológica del suelo es muy sensible a los disturbios ocasionados por el laboreo. Se evaluaron los cambios de la condición biológica originados por el escarificado en un suelo bajo siembra directa estabilizada. Esta investigación se condujo en un cultivo de maíz en Zavalla, Argentina $\left(33^{\circ} 01^{\prime} \mathrm{S}, 60^{\circ} 53^{\prime} \mathrm{O}\right)$, en un suelo Argiudol vértico. Los tratamientos fueron siembra directa y siembra directa escarificada. Se determinaron carbono orgánico, nitratos, sulfatos y fósforo disponibles; biomasa y actividad respiratoria microbiana, propiedades de la meso y macrofauna, galerías de raíces, presencia de hongos, de materia orgánica en descomposición, krotovinas y profundidad del sistema radical a la quinta hoja del cultivo, y a la cosecha, materia seca aérea y radical. Los datos se procesaron mediante análisis uni y multivariado. La descompactación originada por la labranza profunda incidió en la condición biológica del suelo bajo siembra directa. El escarificado promovió el desarrollo del sistema radical sin impactar significativamente en la producción física del maíz. Creó un edáfico ambiente que condicionó los procesos reguladores de la población microbiana estimulando su actividad y la dinámica de la materia orgánica sin modificar sustancialmente la fauna del suelo establecida durante el periodo de implementación de la siembra directa. El análisis multivariado resultó una herramienta adecuada de evaluación de estos cambios.

Palabras clave: Condición biológica, paratill, siembra directa, Zea mays.

\section{Literatura citada}

Azooz, R.H., B. Lowery y T. Daniel. 1995. Tillage and residue management influence on corn growth. Soil and Tillage Research 33:215-227.

Abu Hamdeh, N.H. 2003. Compaction and subsoling effects on corn growth and soil bulk density. Soil Sci. Soc. Am. J. 67:1213-1219.

Altier N., N. Bajsa, G. Azziz, L. Quagliotto, S. Zerbino, A. Morón y A. Arias. 2005. Biodiversidad del suelo bajo diferentes sistemas de producción. XXI Reuniao do Grupo Técnico em Forrageiras do Cone Sul-Grupo Campos. Desaíos e oportunidaes do Bioma Campos frente a Expansao e Intensificacao Agrícola.

Alvarez Castillo, H., A.M. Vinvini, A.N. López, D.M. Carmona y P.L. Manetti. 2005. Composición específica y funcional de la fauna edáfica en agrosistemas experimentales con rotaciones 
mixtas. Ensayo de Rotaciones y Laboratorio de Servicio de Suelo. Instituto Nacional de Tecnología Agropecuaria (INTA). Balcarce y Facultad Ciencias Agrarias, Universidad Nacional de Mar del Plata. 16 pp.

Alvarez, R. y C. Alvarez. 2000. Soil organic matter pool and their association with carbon minerazation kinetics. Soil Sci. Soc. Am J. 64:184-189.

Anderson, J.P. y K.H. Damsch. 1978. A physiological method for the quantitative measurement of microbial biomass in soils. Soil Biol. Biochem. 10:215-221.

Bonel, B., S. Montico, G. Sponda y N. Spurio. 2005. Descompactación del suelo mediante el uso de escarificadores en Argiudoles típicos del sur de Santa Fe. En: Reología de suelos bajo tráfico. $1-11$

Bonel, B, J. Denoia, M. Costanzo, G. Giubileo y G. Zerpa. 2004. Efecto de la labor de escarificado sobre un argiudol vértico bajo siembra directa continua. Ciencia e Investigación Agraria 31:187-196.

Braim, M.A, K. Chaney y D.R. Hodgson. 1984. Preliminary investigation on the response of spring barley (Hordeum sativum) to soil cultivation with the paraplow. Soil Tillage. Res. 4:277-293.

Bray R.H. y L.T. Kutz. 1945. Determination of total, organic and available forms of phosphate in soils. Soil Sci. 59:39-45.

De Cary, R.S. y D. Hervé. 1994. El estado microbiológico del suelo, indicador de una restauración de la fertilidad. Ecología en Bolivia 41:103-115.

Domínguez, J, J.M. Ressia, D. Jorajuria, R.H. Balbuena y G. Mendivil. 2000. Reología del suelo traficado bajo tres diferentes tratamientos mecánicos. En: Avances en Ingeniería Agrícola. Editorial Orientación Gráfica SRL. Buenos Aires, Argentina. 686 pp.

Domínguez, G., G. Studdert, H. Echeverria, y G. Lorenz. 2004. Efectos de dos sistemas de labranzas sobre la materia orgánica en un Molisol de Balcarce. CD del XIX Congreso Argentino de la Ciencia del Suelo. Paraná, Argentina

Ferraro, D.O. 2005. La sustentabilidad agrícola en la Pampa Interior (Argentina): desarrollo y evaluación de indicadores de impacto ambiental del uso de pesticidas y labranzas. Tesis Doctoral. Facultad Agronomía. Universidad Nacional de Buenos Aires, Argentina. 153 pp.

Frioni, L. 2006. Microbiología Básica, Ambiental y Agrícola. Universidad de la República. Uruguay. FAGRO. Departamento de Publicaciones. Montevideo, Uruguay. 464 pp.
Giubileo, G., V. Bisaro y A. Trevisán. 2006. Introducción al diseño y análisis de experimentos. Ed. Juglaría, Rosario. Argentina. $31 \mathrm{pp}$.

Gizzi, A.H., H. Alvarez Castillo, P. Manetti, A. López, N. Clemente y G. Studdert. 2006. Caracterización de la meso y macrofauna edáficas en sistemas de cultivo del sudeste bonaerense. CD XX Congreso Argentino de la Ciencia del Suelo. Salta, Argentina.

Hipps, N.A. y D. R. Hodgson. 1988. Residual effects of slant-legged subsoiler on shoot growth and grain yield of spring barley. J. Agric. Sci. 111:385-391.

Hollander, M. y D. A. Wolfe. 1973. Non parametrical Statistical Methods. John Wiley and Sons. New York, USA. 385 pp.

Ide, G., G. Hofman, C. Ossemerct y M. van Ruymbeke. 1984. Root growth response of winter barley to subsoiling. Soil Till. Res. 4:419-431.

Jackson, M.1964. Análisis Químico de Suelos. Ed. Omega S.A. Barcelona, España. 662 pp.

Jenkinson, D. and D. Powlson. 1976. Effects of biocidal treatments on metabolism in soil. V. A method for measuring the soil biomass Soil Biol. Biochem. 8:209-213.

Karlen, D., M. Mausbach, J. Doran, R. Cline, R. Harris y G. Schuman. 1997. Soil quality: a concept, definition, and framework for evaluation. Soil Sci. Soc. Am. J. 61:4-10.

Karlen, D., C. Ditzler and S. Andrews. 2003. Soil quality. Geoderma 114:145-156.

Kladivko, E. 2003. Proceedings of the Crop Protection Technology Conference, January 78, 2003, University of Illinois. Urbana. USA.

Lavelle, P. 1997. Faunal activities and soil proceses: adaptative strategies that determine ecosystems function. Adv. Ecol. Res. 27:93-132.

Maddonni, G.A., S.Urricariet, C. Ghersa y R. Lavado. 1999. Assessing soil quality in the rolling Pampa, using soil properties and maize characteristics. Agron. J. 91:280-287.

Marasas, M. 2002. La coleopterofauna edáfica y su relación con la calidad del suelo. Capítulo 7. Páginas 135-151. En: Agroecología. El Camino hacia una Agricultura Sustentable. S.J. Sarandon (ed.) Ediciones Científicas Americanas. Buenos Aires. Argentina. 501 pp.

Marelli, H. y J. Arce. 1989. Siembra directa de soja sobre trigo. Páginas 604-614. En: IV Conferencia Mundial de Investigación en Soja. Tomo II. Buenos Aires. Argentina.

Martino, D.L. 1998. Manejo de restricciones físicas del suelo en sistemas de siembra directa. Pages 22. In: Alleviation of soil physical constraints in direct-seeding systems in Uruguay. Ph.D. 
Thesis. University of Manitoba, Manitoba, Canada.

Nelson, D.W. y L.E. Sommers. 1996. Total carbon, organic carbon, and organic matter. p. 9611009. In Sparks D.L., and J.M. Bartels (eds.). Methods of Soil Analysis: Chemical Methods. Part 3. SSSA Book Series number 5. Madison, Wisconsin, USA.

Nolin, M.C., Wang, C. y Caillier, M.J. 1989. Based on selected soil characteristics of the plow layer. Can. J. Soil Sci. 69:525-541.

Orchard, V. y J.Cook. 1983. Relationship between soil respiration and soil moisture. Soil Biol. Biochem 15:447-453.

Paul, E.,D.Harris, H. Collins, U.Schultess y G.Robertson.1999. Evolution $\mathrm{CO}_{2}$ and soil carbon dynamics in biologically managed, rowcrop agroecosystems. Applied Soil Ecol. 11:5365.

Pires da Silva, A, S. Imhoff, N.Giarola y C. Tormena. 2001. Análisis multivariado y univariado en la discriminación de sistemas de uso de suelos del centro de Santa Fe. Sociedad Española de la Ciencia del Suelo 8:21-34.

Pikull, J., J..Zuzel y R. Ramig. 1990. Effect of tillage-induced soil macroporosity on water infiltration. Soil Tillage Research 17:153-165.

Quiroga, A.R., D.E. Buschiazzio y N. Peinemann. 1998. Management discriminant properties in semiarid soils. Soil Sci. 163:591-597.

Romaniuk, R., A. Costantini y L. Giuffré. 2006. Evaluación de parámetros biológicos como potenciales indicadores calidad del suelo. CD Congreso Ciencia del Suelo. Salta. Argentina.

Sacchi, O., M. Costanzo y A. Coronel. 2002. Caracterización de las precipitaciones en la Localidad de Zavalla. Rev. Inv. Fac. Cs. Agr, Universidad Nacional de Rosario. 2:91-103.

Shapiro, S. y M.Wilk. 1965. An analysis of variance test for normality. Biometrika. 52:591-611.
Singh, J. y S. Gupta. 1997. Plant decomposition and soil respiration in terrestrial ecosystems. The Botanical Review. 43:449-523.

Sylvia,D., J. Fuhrmann, P. Hartel y D. Zuverer. 1999. Principles and Applications of Soil Microbiology. Prentice Hall.Upper Saddle iver, NJ, USA. $550 \mathrm{pp}$.

Tabatabai, M.A. 1982. Sulfur. In: A. Payne, J. Freney, and R. Miller (eds.). Methods of Soil Analysis. Part 2: Chemical Properties. $2^{\circ}$ ed., ASA-SSSA, Madison, USA. 1159 pp.

Tate III, R. 1995. Soil microbiology. John Wiley and Sons, NY, USA. 398 pp.

Torella J., E. Angelotti, R. Introcaso, J. Ceriani y J. Guecaimburu. 2004. Respuesta de parámetros biológicos del suelo a la implementación de siembra directa. CD XIX Congreso Argentino de la Ciencia del Suelo. Paraná. Argentina. 10 pp

Vilche, M.S., J. Denoia, S. Montico, B. Tonel y N. Dileo. 2006. El uso de la energía en los sistemas agropecuarios del distrito Zavalla (Santa FE). RevIsta Científica Agropecuaria 10:7-19.

Vilche, M. C.Alzugaray y S. Montico. 2002. Efecto de la labranza y duración de las praderas sobre la condición física de un suelo argiudol vértico de Argentina. Revista Ciencia e Investigación Agraria 29:159-169.

Wander, M. y Bolero, G.A.. 1999. Soil quality assesment of tillage impacts in Illinois. Soil Sci. Soc. Am. J. 63:961-971.

Ward, J.1963. Hierarchical grouping to optimize and objective function. American Statistical Association Journal 58:236-244.

Zerbino, S. y N. Altier. 2006. La Biodiversidad del suelo. 8-9. En: Suplemento Tecnológico. Instituto Nacional de Investigaciones Agropecuarias (INIA), La Estanzuela. Uruguay. 